\title{
Trajectory estimation for fresh impacts on Mars
}

\author{
E.D. Podobnaya, O.P. Popova and D.O. Glazachev
}

Sadovsky Institute of Geosphere Dynamics

Leninsky prospect 38, Moscow, Russia, (E-mail: epodobnaya@gmail.com)

Received: August 1, 2021; Accepted: November 10, 2021

\begin{abstract}
Exploration of small craters and craters clusters on Mars allows one to study meteoroid fragmentation details that cannot be detected in terrestrial conditions. It was suggested earlier that the description of a cluster with a scattering ellipse allows one to estimate the meteoroids' trajectory, which is connected with orbital parameters of the impactor. Independent construction of scattering ellipses and comparison with crater ejecta demonstrate the accuracy and find out some problems of this approach.
\end{abstract}

Key words: meteoroids - Mars - craters - impacts - cluster - fragmentation - strewn fields

\section{Introduction}

In recent years, about 700 fresh dated meteoroid impact sites were discovered on Mars (Malin et al., 2006; Daubar et al., 2013, 2019). Meteoroid impacts resulted in the formation of single craters and crater fields, with crater sizes up to $50 \mathrm{~m}$.

Due to the more rarefied Mars atmosphere (in comparison with Earth) falling meteoroids are less destroyed. Nevertheless, near half of meteoroids are fragmented in the Martian atmosphere and are forming crater clusters (Daubar et al., 2013, 2019).The comparison of Martian and terrestrial fireballs allows to suggest $40-50 \%$ of terrestrial meteoroids would lead to the formation of crater fields on Mars (Hartmann et al., 2018). On Earth, meteoroids are usually observed during a short flight through the atmosphere. In rare cases, their fragments are found as meteorites. Estimates meteoroids properties depend on used fragmentation models calibrated on a small number of events. As Mars surface level corresponds to about $30 \mathrm{~km}$ altitude in the Earth atmosphere, crater scattering fields on Mars surface provide a unique opportunity to see the results of less significant fragmentation.

Detailed data for 77 crater fields (clusters) containing from 2 to 465 individual craters, were obtained by HiRISE camera (High Resolution Imaging Science Experiment), which made high resolution images of Mars (Daubar et al., 2013, 2019). The minimum size of craters in clusters is about $1 \mathrm{~m}$ due to the resolution of the cameras. The average diameter of craters in various clusters is from 1 to $6 \mathrm{~m}$, the average value for all clusters is $2.5 \mathrm{~m}$. Clusters craters (Fig. 1) can be de- 


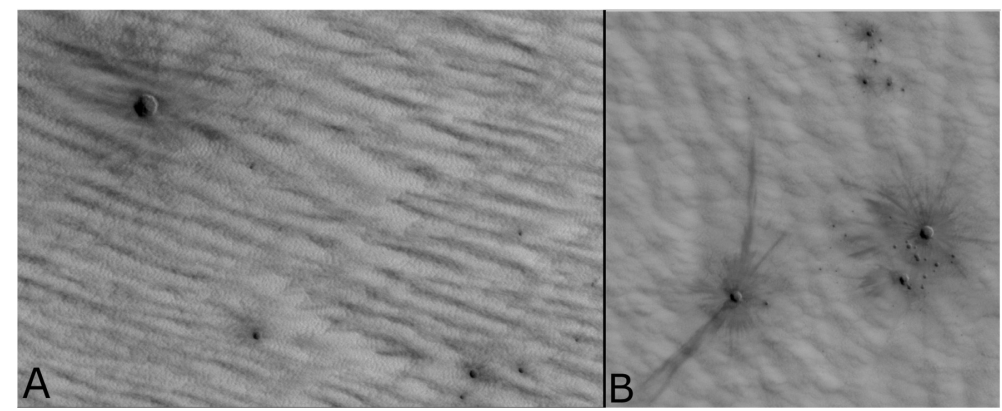

Figure 1. HiRISE images for craters distribution in two clusters under study. A: Cluster ESP_013655_1710. B: Cluster ESP_013800_1820.

scribed by the scattering ellipse, which allows to estimate the parameters of the meteoroid trajectory, for example azimuth and entry angle into the atmosphere.

Destruction of a space object can be a rather complex, multi-stage process. The description of the meteorites scattering field by an ellipse was initially proposed for the case of a single fragmentation. Scattering ellipse parameters depend on the trajectory of the multiply fragmented space object, although possibly in a more complex way. A closer look at the location of the craters within the scattering ellipses may reveal more about the fragmentation process and impactor properties. The size of cluster depends on many factors, including the size of the impactor, its strength and the strength of its fragments, density, the fragmentation process, and others (Ivanov et al., 2008). Cluster study can provide an opportunity to determine these characteristics. Finding the smallest ellipse fitting the cluster is one of the first approaches to describe clusters (Daubar et al., 2019).

\section{Scattering ellipses}

To describe clusters and obtain various estimates (entry angle, azimuth), Daubar et al. (2019) proposed to fit the minimum ellipse covering $90 \%$ of the craters in the cluster. Here scattering ellipses were constructed for 55 clusters, which contain more than 5 craters. It is not clear how sensitive the estimates of the angles and cluster sizes obtained by Daubar et al. (2019) are to the applied method, so we used the same algorithm independently. In addition, we tested several more algorithms and chose the best of them (Podobnaya et al., 2020). First algorithm, called below MVE, is similar to the method applied by Daubar et al. (2019). Minimal square ellipses, which cover 300 random sets of craters (with possible duplication), were built for each cluster. Bootstrap procedure was used for more reliable results and possible incomplete data compensation. Averaged ellipse is used as the result. Next algorithm, called below Stat, is a 
statistical ellipse, implemented in Wolfram Mathematica software. Constructed scattering ellipses (Fig. 2) differ from the independent source (Daubar et al., 2019) by ellipse area up to 1.5 times.

The ratio of the ellipse semi-axes was used to determine the meteoroids entry angle. Our estimates made by two methods mentioned above differ by no more than $15^{\circ}$ in most cases. Obtained trajectory projection angle (i.e. azimuth without direction) demonstrates not more than $20^{\circ}$ difference between MVE, Stat and an independent estimate (Daubar et al., 2019) in most cases. The direction of flight of the meteoroid (azimuth) was determined as the direction from the center of the scattering ellipse along its major axis towards the maximum crater. Azimuths and other angles in this work are counted clockwise from north. The flight direction coincides with an accuracy of $45^{\circ}$ in about $80 \%$ of cases, and in $10-15 \%$ the direction is opposite (depending on the algorithm of the scattering ellipse constructing). In the remaining $5-10 \%$ of cases, the main axis of the ellipses is perpendicular to those indicated in Daubar et al. (2019).

\section{Scattering ellipses on Earth}

As an independent testing, the considered methods of constructing a scattering ellipse (MVE, Stat) were applied to two recent terrestrial scattering fields the Ozerki and Chelyabinsk meteorites, for which all trajectory parameters are known (Kartashova et al., 2020; Borovička et al., 2013; Popova et al., 2013). The angle of entry into the atmosphere and azimuth were found from the obtained scattering ellipses.

In the case of the Chelyabinsk meteoroid, data of 180 fragments (the maximum fragment weight is $600 \mathrm{~kg}$ ) were considered (Popova et al., 2013). The obtained estimates of the flight direction coincide with the results obtained by other methods - 283-284 (Popova et al., 2013; Borovička et al., 2013) with an accuracy of several degrees (Fig. 3). For the entry angle into the atmosphere of the Chelyabinsk meteoroid considering scattering ellipses give the angle of $7-8^{\circ}$, with a known estimate of $18^{\circ}$ (the angle is measured from the horizontal) (Popova et al., 2013). According to the data of the nearest meteorological stations (Verkhneye Dubrovo, $180 \mathrm{~km}$ from Chelyabinsk), the wind speed during the meteoroid flight at altitudes below $20 \mathrm{~km}$ did not exceed $15 \mathrm{~m} \mathrm{~s}^{-1}$, and the wind direction was approximately $320-330^{\circ}$ (Fig. 3 ). The difference in the estimates relate to wind influence on small fragments and with incomplete collection of small fragments, the real scattering field was probably wider.

The scattering ellipses obtained from the data of 76 fragments (the maximum fragment mass is $740 \mathrm{~g}$ ) of the Ozerki meteorite (Popova et al., 2019) were compared with the trajectory found in Kartashova et al. (2020). Our estimates of meteoroid entry angle into the atmosphere $\left(33-35^{\circ}\right)$ correlate poorly with the estimate at $77.6^{\circ}$ (from the horizon) (Kartashova et al., 2020). The azimuth of the Ozerki meteoroid flight is also estimated based on the obtained scattering 


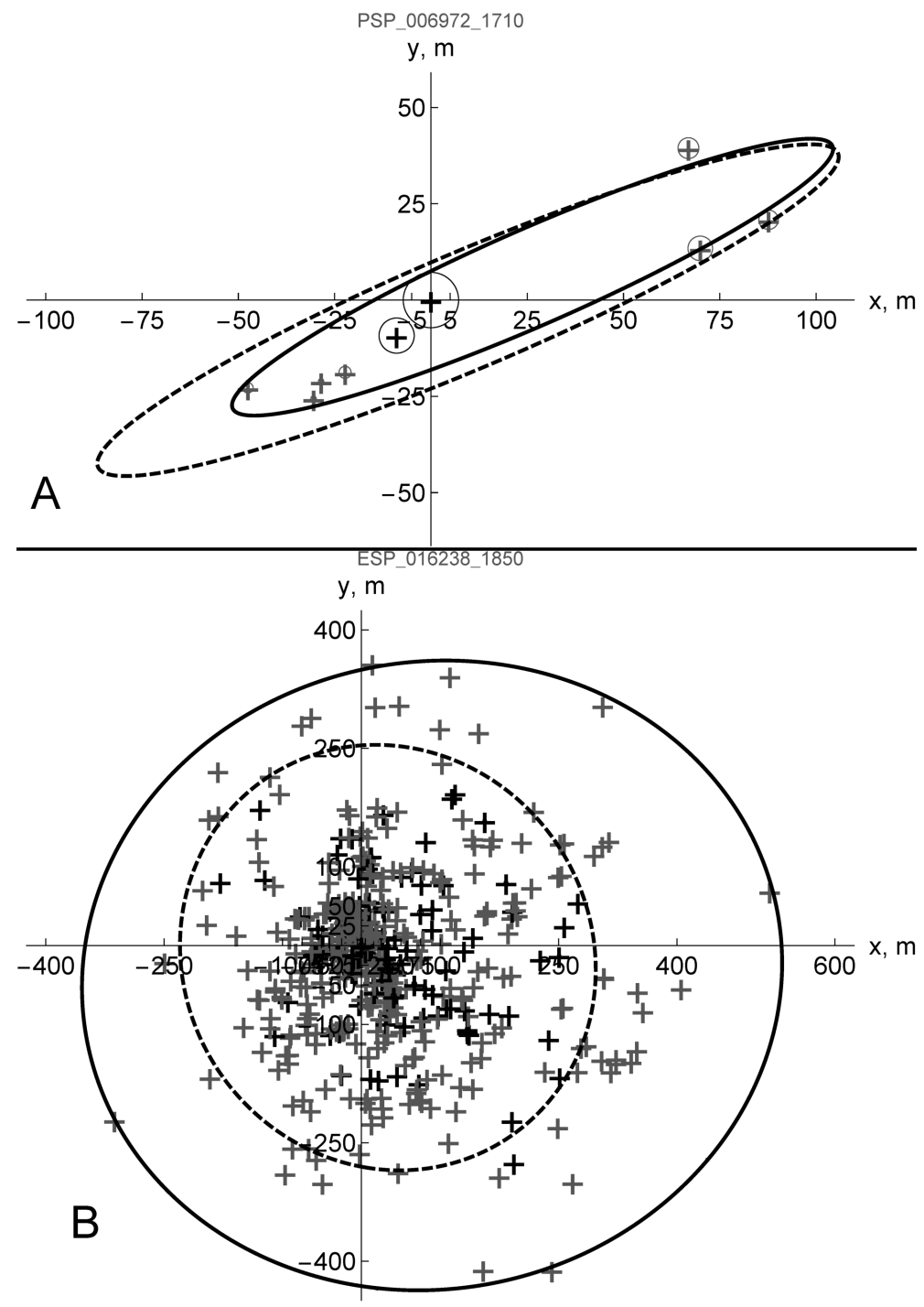

Figure 2. Minimal square ellipses covering $90 \%$ of the craters in the cluster, constructed in two different ways. Solid line corresponds to MVE ellipse and dashed to Stat one. Pluses show the location of crater centers inside the cluster, the circles around show the size of craters (ones marked black are included in upper quantile by size). A: Cluster PSP_006972_1710 with small number of craters (N=9). B: Cluster ESP_016238_1850 with large number of craters $(\mathrm{N}=465)$. 


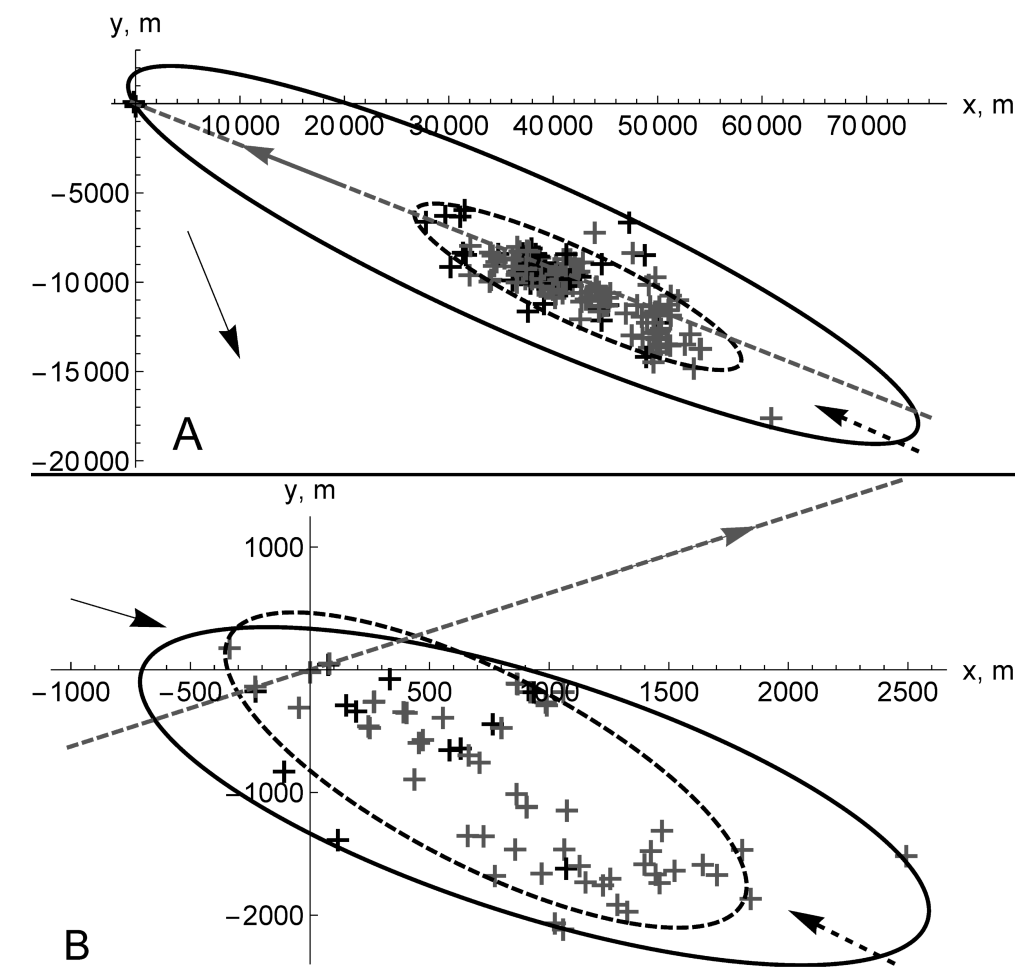

Figure 3. Scattering ellipses on the Earth covering the found fragments and constructed by two different methods. Solid line corresponds to MVE ellipse and dashed - to Stat. Pluses show the location of fragments inside the cluster (black ones are included in upper quantile by size). The black solid arrow marks the wind direction. A: Chelyabinsk meteorite. The dashed line indicates the trajectory from an independent source (Popova et al., 2013), which coincides with one obtained in this work (the arrow on the trajectory line shows the direction of flight). B: Meteorite Ozerki. The dashed line refer to the trajectory from an independent source (Kartashova et al., 2020) (the arrow on the trajectory line shows the direction of flight). The dashed arrow indicates the average trajectory and direction of flight for discussed methods.

ellipses as $308-321^{\circ}$ (taking into account the direction; Fig. 3). Analysis of video records (Kartashova et al., 2020) results in azimuth about $58^{\circ} \pm 3^{\circ}$ (the trajectory is directed from southwest to northeast; Fig. 3), the difference with our estimates is large. According to the data of the nearest meteorological station $(50 \mathrm{~km})$, the wind during the meteoroid flight was directed from an azimuth of $300^{\circ}$ at altitudes below $20 \mathrm{~km}$. Based on the location of small fragments to the right of the ellipse (Fig. 3), it can be concluded that the positions of small fragments (less than $1 \mathrm{~kg}$ ) are primarily determined by the wind. The scattering 
field of the Ozerki meteorite is elongated in the direction of the wind; for small fragments with an almost vertical entry into the atmosphere, it is primarily determined by the wind.

\section{Ejecta}

The crater ejecta gives the possibility to estimate the direction of the flight in some cases. For oblique impacts crater ejecta is asymmetrical and is more pronounced in the direction of flight (Shuvalov, 2011). The images allow us to consider craters ejecta in detail for a number of clusters. The direction of flight determined by the ejecta was compared with estimates from scattering ellipses. The azimuths were found based on the location of craters ejecta from the images of Mars provided by the HiRISE project (Burleigh et al., 2012; Ivanov et al., 2010). The azimuth estimates for the projection of fragments trajectory from crater ejecta were done for 41 out of 55 considered clusters, examples of comparing meteoroid trajectory for two clusters are shown at Fig. 4. Flight direction of the meteoroid obtained by the crater ejecta does not agree very well with the azimuths calculated from the scattering ellipses. In one third of the cases the difference is no more than $45^{\circ}$, in $40 \%$ of the cases the results differ in direction $\left(180^{\circ} \pm 45^{\circ}\right)$. For $30 \%$ of cases the directions are perpendicular, i.e., ejecta is directed mainly along the minor axis of the scattering ellipse (i.e., across the scattering field).

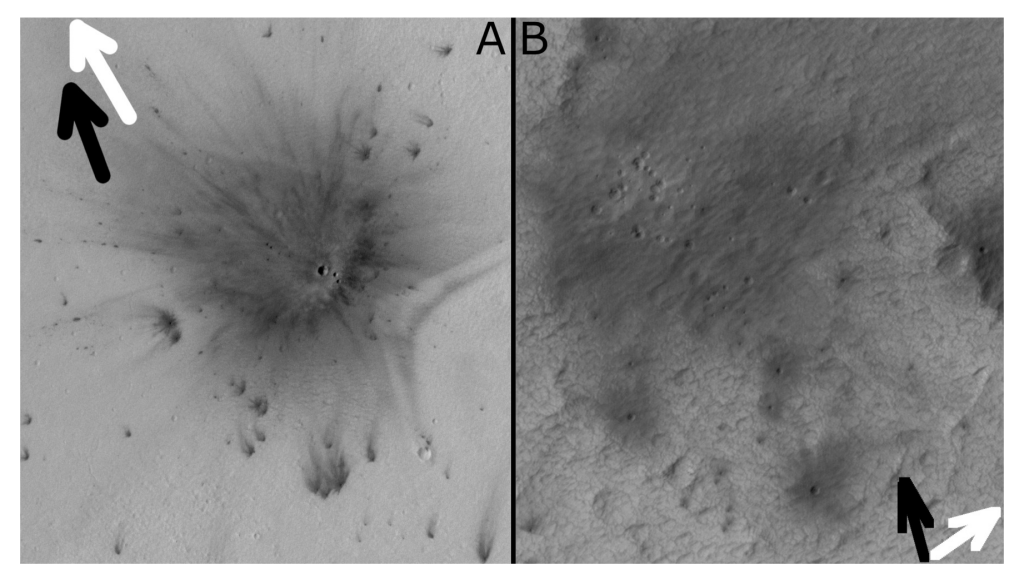

Figure 4. Images from HiRISE for two clusters. Arrows show meteoroid flight direction obtained by scattering ellipse (black arrows) and by craters ejecta location (white arrows). A: Cluster ESP_024646_1890. B: Cluster ESP_026009_1920. 


\section{Summary}

Crater strewn fields are often described by an ellipse. It is suggested that this description allows to estimate trajectory azimuth and entry angle based on the scattering ellipse. Several methods of ellipse construction were applied to fresh Martian crater clusters and best ones were chosen. The resulting ellipses were compared with each other and with an independent estimate. Comparison (by the number of covered craters and by area) showed that the considered methods give a scattering ellipse with an area of 1.41.7 times to the area obtained from the initial estimates (Daubar et al., 2019) and cover at least $88 \%$ of craters in clusters in average. From the scattering ellipses, estimates of the parameters for meteoroids entry into the atmosphere (entry angle and azimuth) were obtained. In general, all the considered methods give approximately the same value of the meteoroid trajectory azimuth, the difference in most cases does not exceed $15^{\circ}$. Meteoroid flight is directed from the center of the scattering ellipse along its major axis towards the maximum crater. The flight direction coincides with an accuracy of $45^{\circ}$ in $80 \%$ of cases, in $10-15 \%$ - the direction is opposite. In the remaining $5-10 \%$ of cases the constructed scattering ellipses are perpendicular to the independent source estimates (Daubar et al., 2019). The meteoroid entry angle into the atmosphere depends on the ratio of the scattering ellipse semiaxes; its estimates by different methods in most cases differ by less than $15^{\circ}$ from the initial estimate (Daubar et al., 2019).

The algorithms for constructing scattering ellipses were applied to the data on the fragmentation of two meteoroids on Earth - the Ozerki and Chelyabinsk meteorites. It was shown that it is possible to estimate the azimuth and the entry angle for large fragments in oblique impact, whose fall is little affected by wind. The scattering field of small fragments of a meteoroid with an almost vertical trajectory of entry is determined mainly by wind drift.

For some clusters, the azimuth of the meteoroid trajectory was found from craters ejecta. In about $70 \%$ of these clusters the ejecta is oriented along the scattering ellipse major axis (with accuracy $45^{\circ}$ ), the direction of flight coincides only in $30 \%$. The direction of the ejecta from the crater along the minor axis of the scattering ellipse, obtained in $30 \%$ of cases, requires consideration. Modeling the formation of scattering fields probably help to solve these problems.

\section{References}

Borovička, J., Spurný, P., Brown, P., et al., The trajectory, structure and origin of the Chelyabinsk asteroidal impactor. 2013, Nature, 503, 235, DOI: 10.1038 /nature 12671

Burleigh, K. J., Melosh, H. J., Tornabene, L. L., et al., Impact airblast triggers dust avalanches on Mars. 2012, Icarus, 217, 194, DOI: 10.1016/j.icarus.2011.10.026 
Daubar, I. J., Banks, M. E., Schmerr, N. C., \& Golombek, M. P., Recently Formed Crater Clusters on Mars. 2019, Journal of Geophysical Research (Planets), 124, 958, DOI: 10.1029/2018JE005857

Daubar, I. J., McEwen, A. S., Byrne, S., Kennedy, M. R., \& Ivanov, B., The current martian cratering rate. 2013, Icarus, 225, 506, DOI: 10.1016/j.icarus.2013.04.009

Hartmann, W. K., Daubar, I. J., Popova, O., \& Joseph, E. C. S., Martian cratering 12. Utilizing primary crater clusters to study crater populations and meteoroid properties. 2018, Met. Planet. Sci., 53, 672, DOI: 10.1111/maps.13042

Ivanov, B. A., Melosh, H. J., McEwen, A. S., \& HiRISE Team, Small Impact Crater Clusters in High Resolution HiRISE Images. 2008, in Lunar and Planetary Science Conference, Lunar and Planetary Science Conference, 1221

Ivanov, B. A., Melosh, H. J., McEwen, A. S., \& HiRISE Team, New Small Impact Craters in High Resolution HiRISE Images - III. 2010, in Lunar and Planetary Science Conference, Lunar and Planetary Science Conference, 2020

Kartashova, A., Golubaev, A., Mozgova, A., et al., Investigation of the Ozerki meteoroid parameters. 2020, Planetary Space Science, 193, 105034, DOI: 10.1016/j.pss.2020.105034

Malin, M., Edgett, K., Posiolova, L., McColley, S., \& Noe, D. E., Catalog of new impact sites on Mars formed May 1999-March 2006. Malin Space Science Systems. 2006, Inc., San Diego, California

Podobnaya, E., Popova, O., \& Glazachev, D., Scattering Ellipses for Fresh Clusters of Craters on Mars. 2020, Collection of scientific papers IDG RAS. Dynamic processes in geospheres. Issue 12. (In Russian)

Popova, O., Rybnov, Y., Kharlamov, V., \& Glazachev, D., Analysis of Optical and Infrasonic Observations of the Ozerki Meteorite Fall. 2019, Abstract Book of the 5th International Conference "Trigger Effects in Geosystems", 165

Popova, O. P., Jenniskens, P., Emel'yanenko, V., et al., Chelyabinsk Airburst, Damage Assessment, Meteorite Recovery, and Characterization. 2013, Science, 342, 1069, DOI: 10.1126/science. 1242642

Shuvalov, V., Ejecta deposition after oblique impacts: An influence of impact scale. 2011, Met. Planet. Sci., 46, 1713, DOI: 10.1111/j.19455100.2011.01259.x 